\title{
QUASI-INTERPOLANTS SPLINES : EXEMPLES ET APPLICATIONS
}

\author{
PAUl SABLONNiÈre ${ }^{1}$
}

\begin{abstract}
A quasi-interpolant (abbr. QI) is an approximation operator obtained as a finite linear combination of basis functions with bounded support (B-splines). In addition, the coefficient of a B-spline is a linear functional (of differential, discrete or integral type) acting on the function to be approximated in a neighbourhood of the support of that B-spline. The big advantage of this approach is that the computation of a QI is direct and does not need the solution of any system of equations, unlike what happens with interpolants. It is particularly interesting in the bivariate or trivariate cases, where the number of B-splines can be rather large. In this paper, I present some examples of QIs of different types on spaces of univariate or multivariate splines. Then, I give some applications to approximation and numerical analysis.
\end{abstract}

Résumé. Un quasi-interpolant spline (abréviation QI) est un opérateur d'approximation obtenu comme combinaison linéaire de fonctions à support borné (B-splines) :

$$
Q f=\sum_{\alpha \in A} \mu_{\alpha}(f) B_{\alpha}
$$

Le coefficient $\mu_{\alpha}(f)$ de la B-spline $B_{\alpha}$ est une forme linéaire agissant sur la fonction $f$ à approcher dans un voisinage du support de $B_{\alpha}$. Le grand avantage de cette approche est que le calcul d'un QI est direct et ne nécessite pas la résolution d'un système d'équations, contrairement à ce qui se passe pour un interpolant. C'est particulièrement intéressant en dimension 2 ou 3 , où le nombre de B-splines peut être relativement grand. Dans cet article, je décris quelques exemples de QIs de différents types sur des espaces de splines à une ou deux variables. Puis je présente quelques applications en approximation et en analyse numérique.

\section{InTRODUCTION, DÉFINITIONS ET NOTATIONS}

Soit $\mathbb{F}$ un espace de fonctions, $\mathbb{S}$ un espace de splines polynômiales d'une ou plusieurs variables et $\mathbb{P}_{n}$ l'espace des polynômes de degré total au plus égal à $n$. On suppose que $\mathbb{P}_{n} \subset \mathbb{S}$ et $\mathbb{P}_{n} \subset \mathbb{F}$. On suppose aussi que $\mathbb{S}$ est engendré par la famille de B-splines $\mathcal{B}=\left\{B_{\alpha}, \alpha \in A\right\}$. Pour tout $\alpha \in A, B_{\alpha}$ est une fonction à support borné désigné par $\operatorname{supp}\left(B_{\alpha}\right)$. La famille $A$ des indices est finie ou infinie suivant que l'on travaille sur un domaine borné ou sur l'espace entier.

\footnotetext{
1 Paul Sablonnière, Centre de mathématiques,

INSA de Rennes, 20 avenue des Buttes de Coësmes, CS 14315,

35043 Rennes cédex, France.

e-mail : psablonn@insa-rennes.fr
} 
Définition 1.1. Un quasi-interpolant (abr. QI) est un opérateur $Q: \mathbb{F} \rightarrow \mathbb{S}$ de la forme

$$
Q f=\sum_{\alpha \in A} \mu_{\alpha}(f) B_{\alpha}
$$

ayant les propriétés suivantes :

(1) $Q$ est un opérateur exact sur $\mathbb{P}_{n}: Q p=p, \forall p \in \mathbb{P}_{n}$.

(2) $Q$ est un opérateur local : le coefficient $\mu_{\alpha}(f)$ est une forme linéaire qui ne dépend que des valeurs de $f$ dans un voisinage de $\operatorname{supp}\left(B_{\alpha}\right)$.

Voici le plan de l'article : dans la première partie, je donne différents exemples de quasi-interpolants splines en dimension 1 et 2. Je me limite ici aux QI quadratiques de classe $C^{1}$ sur une partition uniforme de la droite ou du plan, afin de simplifier la présentation. Mais il est possible de définir des QI de tous les degrés sur une subdivision quelconque d'un intervalle arbitraire en dimension 1. En dimension 2, on peut aussi définir des QI de tous degrés sur certaines triangulations. Je renvoie aux références (section 8) pour de plus amples informations. Dans la deuxième partie, je donne quelques applications des quasi-interpolants quadratiques, d'abord en dimension 2 à l'approximation d'une fonction et de ses dérivées partielles et à la recherche de ses points stationnaires, puis en dimension 1 à l'intégration et à la dérivation approchées d'une fonction, à une méthode pseudo-spectrale pour la résolution approchée d'une équation différentielle, et enfin à l'approximation des zéros d'une fonction.

\section{QUASI-INTERPOLANT SPLINES QUADRATIQUES SUR $\mathbb{R}$}

Soit $\mathbb{F}=C^{2}(\mathbb{R})$ et $\mathbb{S}=S_{2}^{1}(\mathbb{R}, \tau)$ l'espace des splines quadratiques de classe $C^{1}$ sur la subdivision uniforme $\tau=\mathbb{Z}+\frac{1}{2}$. Une fonction de $\mathbb{S}$ est dans $C^{1}(\mathbb{R})$ et sa restriction à chaque intervalle $\left[k-\frac{1}{2}, k+\frac{1}{2}\right]$ est dans $\mathbb{P}_{2}$. Désignons par $B(x)$ la $B$-spline quadratique de support $\left[-\frac{3}{2}, \frac{3}{2}\right]$ centré en 0 , et posons $B_{k}(x)=B(x-k)$ : on a donc $\operatorname{supp}\left(B_{k}\right)=\left[k-\frac{3}{2}, k+\frac{3}{2}\right]$. Schoenberg [11] a démontré que toute spline quadratique $g \in \mathbb{S}$ s'écrit sous la forme :

$$
g(x)=\sum_{k \in \mathbb{Z}}\left(g(k)-\frac{1}{8} D^{2} g(k)\right) B_{k}(x) .
$$

On a bien sûr $\mathbb{P}_{2} \subset \mathbb{S}$ et les premiers monômes (notés $e_{r}(x)=x^{r}, \forall r \geq 0$ ) s'expriment de la manière suivante dans la base des B-splines

$$
e_{0}(x)=1=\sum_{k \in \mathbb{Z}} B_{k}(x), \quad e_{1}(x)=x=\sum_{k \in \mathbb{Z}} k B_{k}(x), \quad e_{2}(x)=x^{2}=\sum_{k \in \mathbb{Z}}\left(k^{2}-\frac{1}{4}\right) B_{k}(x) .
$$

\subsection{Quasi-interpolant discret}

D'après (1), on peut définir un premier opérateur $Q$ de $\mathbb{F}=C^{2}(\mathbb{R})$ dans $\mathbb{S}=S_{2}^{1}(\mathbb{R}, \tau)$ par

$$
Q f(x)=\sum_{k \in \mathbb{Z}}\left(f(k)-\frac{1}{8} D^{2} f(k)\right) B_{k}(x) .
$$

C'est un QI car il est exact sur $\mathbb{P}_{2}$ et la forme linéaire $\mu_{k}(f)=f(k)-\frac{1}{8} D^{2} f(k)$ n'utilise que les valeurs de $f$ et de $D^{2} f$ au point $k$, centre de $\operatorname{supp}\left(B_{k}\right)$. On l'appelle quasi-interpolant différentiel (abr. DQI) et de plus c'est un projecteur sur $\mathbb{S}$ car $Q g=g$ pour tout $g \in \mathbb{S}$.

Une première variante consiste à remplacer $D^{2} f(k)$ par la différence seconde centrée

$$
\delta^{2} f(k)=f(k-1)-2 f(k)+f(k+1) .
$$


On obtient ainsi un second opérateur $Q^{*}$ de $\mathbb{F}=C(\mathbb{R})$ dans $\mathbb{S}=S_{2}^{1}(\mathbb{R}, \tau)$ :

$$
Q^{*} f(x)=\sum_{k \in \mathbb{Z}}\left(f(k)-\frac{1}{8} \delta^{2} f(k)\right) B_{k}(x)=\sum_{k \in \mathbb{Z}} \mu_{k}^{*}(f) B_{k}(x),
$$

où

$$
\mu_{k}^{*}(f)=\frac{1}{8}(-f(k-1)+10 f(k)-f(k+1)) .
$$

Comme $D^{2}$ coïncide avec $\delta^{2}$ sur $\mathbb{P}_{2}, Q^{*}$ est aussi exact sur $\mathbb{P}_{2}$ et c'est un opérateur local car $\mu_{k}^{*}(f)$ n'utilise que les valeurs de $f$ aux trois points entiers de $\operatorname{supp}\left(B_{k}\right)$, mais ce n'est plus un projecteur sur $\mathbb{S}$. On l'appelle quasi-interpolant discret (abr. dQI).

On peut calculer exactement la norme infinie de $Q^{*}$. Il suffit d'écrire $Q^{*} f$ sous la forme :

$$
Q^{*} f(x)=\sum_{k \in \mathbb{Z}} f(k) \tilde{B}_{k}(x)
$$

où $\tilde{B}_{k}$ désigne la fonction fondamentale

$$
\tilde{B}_{k}=\frac{1}{8}\left(-B_{k-1}+10 B_{k}-B_{k+1}\right) .
$$

On montre alors facilement que $\left\|Q^{*}\right\|_{\infty}$ est égale à la norme de Tchebychev de la fonction de Lebesgue $\Lambda^{*}$ de l'opérateur :

Par un calcul simple, on obtient

$$
\left\|Q^{*}\right\|_{\infty}=\left|\Lambda^{*}\right|_{\infty}, \quad \text { où } \quad \Lambda^{*}=\sum_{k \in \mathbb{Z}}\left|\tilde{B}_{k}\right| .
$$

Avec les notations suivantes :

$$
\left\|Q^{*}\right\|_{\infty}=\frac{5}{4}=1.25
$$

$$
|g|_{\infty, k}=\max \left\{|g(x)|, x \in\left[k-\frac{1}{2}, k+\frac{1}{2}\right]\right\} \quad \text { et } d_{k}\left(f, \mathbb{P}_{2}\right)=\inf \left\{|f-p|_{\infty, k}: p \in \mathbb{P}_{2}\right\}
$$

on montre (voir par exemple [5]) que pour tout $f \in C(\mathbb{R})$ et tout $k \in \mathbb{Z}$ :

$$
\left|Q^{*} f-f\right|_{\infty, k} \leq\left(1+\left\|Q^{*}\right\|_{\infty}\right) d_{k}\left(f, \mathbb{P}_{2}\right)=2.25 d_{k}\left(f, \mathbb{P}_{2}\right)
$$

Ceci montre que $Q^{*} f$ est très proche de la meilleure approximation polynômiale de $f$ dans chaque intervalle. On en déduit que sur la subdivision $h \mathbb{Z}$, on a, pour une fonction $f \in C^{3}(\mathbb{R})$,

$$
\left|Q^{*} f-f\right|_{\infty}=O\left(h^{3}\right) .
$$

\subsection{Quasi-interpolant intégral}

On va définir maintenant une 2ème variante du quasi-interpolant $Q$. Pour cela, on utilise les trois premiers moments [41] de la B-spline quadratique $B_{k}$ :

$$
m_{k}^{(0)}=\int_{\mathbb{R}} B_{k}(x) d x=1, \quad m_{k}^{(1)}=\int_{\mathbb{R}} x B_{k}(x) d x=k, \quad m_{k}^{(2)}=\int_{\mathbb{R}} x^{2} B_{k}(x) d x=k^{2}+\frac{1}{4} .
$$

Soit $\langle f, g\rangle=\int_{\mathbb{R}} f(x) g(x) d x$ et soit $\bar{Q}$ le QI intégral suivant :

$$
\bar{Q} f=\sum_{k \in \mathbb{Z}}\left\langle f, \bar{B}_{k}\right\rangle B_{k}
$$


où $\bar{B}_{k}=\frac{1}{4}\left(-B_{k-1}+6 B_{k}-B_{k+1}\right)$. On vérifie facilement que $\bar{Q} e_{r}=e_{r}$ pour $r=0,1$. Comme $\left\langle e_{2}, \bar{B}_{k}\right\rangle=$ $\frac{1}{4}\left(-m_{k-1}^{(2)}+6 m_{k}^{(2)}-m_{k+1}^{(2)}\right)=k^{2}-\frac{1}{4}$, d'après (2) on obtient également

$$
\bar{Q} e_{2}=\sum_{k \in \mathbb{Z}}\left(k^{2}-\frac{1}{4}\right) B_{k}=e_{2}
$$

donc $\bar{Q}$ est exact sur $\mathbb{P}_{2}$ et c'est un opérateur local. On l'appelle quasi-interpolant intégral (abr. iQI). On peut également écrire $\bar{Q} f$ sous la forme

$$
\bar{Q} f=\sum_{k \in \mathbb{Z}}\left\langle f, B_{k}\right\rangle \bar{B}_{k}
$$

ce qui montre que l'information sur $f$ est obtenue au moyen des moyennes locales pondérées

$$
\bar{\mu}_{k}(f)=\left\langle f, B_{k}\right\rangle=\int_{k-3 / 2}^{k+3 / 2} f(x) B_{k}(x) d x
$$

En introduisant la fonction de Lebesgue $\bar{\Lambda}=\sum_{k \in \mathbb{Z}}\left|\bar{B}_{k}\right|$, on peut montrer que

$$
\|\bar{Q}\|_{\infty}=|\bar{\Lambda}|_{\infty}=\frac{11}{8}=1.375
$$

On peut aussi approcher $\bar{\mu}_{k}(f)$ par des formules de quadrature de type Gauss associées à la fonction de poids $B_{k}(x)(\mathrm{cf}[7,18,42])$.

\section{QUASI-INTERPOLANTS SPLINES QUADRATIQUES SUR UNE TRIANGULATION UNIFORME DU PLAN}

Soit $\tau$ la triangulation uniforme de type II du plan obtenue en traçant les deux diagonales dans tous les carrés de sommets les points de $\left(\mathbb{Z}+\frac{1}{2}\right)^{2}$. Les centres de ces carrés sont donc les points de $\mathbb{Z}^{2}$. On note $\varepsilon_{1}=(1,0)$ et $\varepsilon_{2}=(0,1)$ les vecteurs unitaires, et l'on pose $\varepsilon_{3}=\varepsilon_{1}+\varepsilon_{2}=(1,1)$ et $\varepsilon_{4}=-\varepsilon_{1}+\varepsilon_{2}=(-1,1)$. Comme les arêtes du réseau sont parallèles à ces directions, on parle de réseau à 4 directions (4-direction mesh). Soit $\mathbb{S}=S_{2}^{1}\left(\mathbb{R}^{2}, \tau\right)$ l'espace des splines quadratiques de classe $C^{1}$ sur la triangulation $\tau$. Il existe une box-spline $B \in \mathbb{S}$ dont le support est l'octogone centré à l'origine de sommets $\left\{ \pm \frac{1}{2}\left(3 \varepsilon_{1} \pm \varepsilon_{2}\right), \pm \frac{1}{2}\left(3 \varepsilon_{2} \pm \varepsilon_{1}\right)\right\}$. L'espace $\mathbb{S}$ est engendré par les translatées entières de $B$ :

$$
\mathcal{B}=\left\{B_{\alpha}(x)=B(x-\alpha) ; \alpha \in \mathbb{Z}^{2}\right\}
$$

On a évidemment $\mathbb{P}_{2} \subset \mathbb{S}$. De plus, $\mathcal{B}$ est une partition de l'unité, $\sum_{\alpha \in \mathbb{Z}^{2}} B_{\alpha}=1$, et $\int B_{\alpha}=1$. En revanche, la famille $\mathcal{B}$ n'est pas linéairement indépendante : cette propriété, qui s'avère assez gênante pour l'interpolation ou l'approximation au sens des moindres carrés, ne joue aucun rôle dans la construction des QI ci-dessous (voir par exemple $[1-3,10,21-23,45,58])$.

\subsection{Quasi-interpolant discret}

Soit $\Delta f$ le laplacien de $f$. Un premier QI différentiel $Q$ ( [10], chapitre VI) est défini par :

$$
Q f=\sum_{\alpha \in \mathbb{Z}^{2}}\left\{f(\alpha)-\frac{1}{8} \Delta f(\alpha)\right\} B_{\alpha}
$$


A partir des représentations des monômes $e_{r s}(x)=x_{1}^{r} x_{2}^{s}$ de $\mathbb{P}_{2}$ en fonction des B-splines :

$$
\begin{gathered}
e_{00}=\sum_{\alpha \in \mathbb{Z}^{2}} B_{\alpha}, \quad e_{10}=\sum_{\alpha \in \mathbb{Z}^{2}} \alpha_{1} B_{\alpha}, \quad e_{01}=\sum_{\alpha \in \mathbb{Z}^{2}} \alpha_{2} B_{\alpha}, \\
e_{11}=\sum_{\alpha \in \mathbb{Z}^{2}} \alpha_{1} \alpha_{2}, B_{\alpha}, \quad e_{20}=\sum_{\alpha \in \mathbb{Z}^{2}}\left(\alpha_{1}^{2}-\frac{1}{4}\right) B_{\alpha}, \quad e_{02}=\sum_{\alpha \in \mathbb{Z}^{2}}\left(\alpha_{2}^{2}-\frac{1}{4}\right) B_{\alpha},
\end{gathered}
$$

on vérifie que $Q$ est exact sur $\mathbb{P}_{2}$, i.e. $Q p=p$ pour tout $p \in \mathbb{P}_{2}$. A partir de ce $\mathrm{QI}$ différentiel, on peut, comme dans le cas d'une variable, définir un QI discret et un QI intégral qui sont aussi exacts sur $\mathbb{P}_{2}$.

Pour construire le QI discret $Q^{*}$, on remplace $\Delta f(\alpha)$ par le schéma aux différences classique à 5 points, centré au point $\alpha$ :

$$
\Delta^{*} f(\alpha)=-4 f(\alpha)+\frac{1}{8} \sum_{i=1}^{2}\left[f\left(\alpha+\varepsilon_{i}\right)+f\left(\alpha-\varepsilon_{i}\right)\right]
$$

qui vérifie

$$
\Delta^{*} p(\alpha)=\Delta p(\alpha), \quad \forall p \in \mathbb{P}_{2}
$$

Le QI discret associé s'écrit alors :

$$
Q^{*} f=\sum_{\alpha \in \mathbb{Z}^{2}}\left\{f(\alpha)-\frac{1}{8} \Delta^{*} f(\alpha)\right\} B_{\alpha}=\sum_{\alpha \in \mathbb{Z}^{2}} \mu_{\alpha}^{*}(f) B_{\alpha}
$$

dont les formes linéaires coefficients sont données par :

$$
\mu_{\alpha}^{*}(f)=\frac{3}{2} f(\alpha)-\frac{1}{8} \sum_{i=1}^{2}\left[f\left(\alpha+\varepsilon_{i}\right)+f\left(\alpha-\varepsilon_{i}\right)\right] .
$$

On démontre que $\left\|Q^{*}\right\|_{\infty}=\frac{3}{2}$, par conséquent l'ordre d'approximation d'une fonction $f$, de classe $C^{3}$ par exemple, est égal à 3 . En effet on a, dans chaque triangle $T$ de la triangulation :

$$
\left|Q^{*} f-f\right|_{\infty, T} \leq\left(1+\left\|Q^{*}\right\|_{\infty}\right) d_{T}\left(f, \mathbb{P}_{2}\right)=\frac{5}{2} d_{T}\left(f, \mathbb{P}_{2}\right)
$$

avec

$$
d_{T}\left(f, \mathbb{P}_{2}\right)=\inf \left\{|f-p|_{\infty, T}: p \in \mathbb{P}_{2}\right\} .
$$

Donc si $\operatorname{diam}(T) \leq h$ pour tout $T \in \tau$ et si $f$ est de classe $C^{3}$, on a

$$
\left|Q^{*} f-f\right|_{\infty}=O\left(h^{3}\right)
$$

On remarque que $Q^{*} f$ est localement très proche de la meilleure approximation de $f$ dans $\mathbb{P}_{2}$. Voir la section 4 ci-dessous pour des résultats plus précis.

\subsection{Quasi-interpolant intégral}

On peut également construire un QI intégral $\bar{Q}$ en utilisant les moments d'ordre $\leq 2$ de la spline $B_{\alpha}(\mathrm{cf}[19])$

$$
\begin{gathered}
m_{\alpha}^{(0,0)}=\int_{\mathbb{R}^{2}} B_{\alpha}=1, \quad m_{\alpha}^{(1,1)}=\int_{\mathbb{R}^{2}} x_{1} x_{2} B_{\alpha}=\alpha_{1} \alpha_{2}, \\
m_{\alpha}^{\varepsilon_{r}}=\int_{\mathbb{R}^{2}} x_{r} B_{\alpha}=\alpha_{r}, \quad m_{\alpha}^{2 \varepsilon_{r}}=\int_{\mathbb{R}^{2}} x_{r}^{2} B_{\alpha}=\alpha_{r}^{2}+\frac{1}{4}, r=1,2 .
\end{gathered}
$$


On définit alors le QI $\bar{Q}$ par :

$$
\bar{Q} f=\sum_{\alpha \in \mathbb{Z}^{2}}\left\langle f, \bar{B}_{\alpha}\right\rangle B_{\alpha}
$$

$\bar{B}_{\alpha}$ étant la fonction définie par

$$
\bar{B}_{\alpha}=2 B_{\alpha}-\frac{1}{4}\left\{B_{\alpha-\varepsilon_{2}}+B_{\alpha-\varepsilon_{1}}+B_{\alpha+\varepsilon_{1}}+B_{\alpha+\varepsilon_{2}}\right\} .
$$

On vérifie alors facilement, en utilisant les représentations (5) et (6) des monômes, que $\bar{Q}$ est exact sur $\mathbb{P}_{2}$ et que $\|\bar{Q}\|_{\infty} \leq 3$. De plus, $\bar{Q}$ est local car le coefficient de $B_{\alpha}$ n'utilise que des moyennes locales pondérées de $f$ dans la réunion des supports des 5 box-splines centrés au point $\alpha$ et en ses 4 voisins immédiats. Ces moyennes sont toutes du type

$$
\int_{\mathbb{R}^{2}} B_{\gamma}(x) f(x) d x
$$

et peuvent être approchées par les formules de quadratures de Gauss associées au poids $B_{\gamma}$. On peut obtenir ainsi de nouvelles familles de QI discrets basés sur les points de Gauss.

\section{Application : Approximation D'Une Fonction de Deux VARiables et de SeS DÉRIVÉES PARTIELLES}

On va utiliser le QI quadratique discret (7) de $f$ sur $\mathbb{R}^{2}$ vu dans la section 3.1 ci-dessus :

$$
\begin{gathered}
Q^{*} f=\sum_{\alpha \in \mathbb{Z}^{2}} \mu_{\alpha}^{*}(f) B_{\alpha} \\
\mu_{\alpha}^{*}(f)=\frac{3}{2} f(\alpha)-\frac{1}{8} \sum_{i=1}^{2}\left[f\left(\alpha+\varepsilon_{i}\right)+f\left(\alpha-\varepsilon_{i}\right)\right] .
\end{gathered}
$$

On sait que $Q^{*}$ est exact sur $\mathbb{P}_{2}$ et que $\|Q\|_{\infty}=1.5$, donc $Q^{*} f$ est très proche localement de la meilleure approximation uniforme de $f$ dans $\mathbb{P}_{2}$. C'est la raison pour laquelle on peut espérer obtenir de bonnes approximations de $f$ et de ses dérivées partielles à partir de ce QI quadratique $C^{1}$ (voir aussi [14] et le numéro spécial dans lequel est publié cet article). La plupart des résultats de cette section proviennent de [30]. Dans ce qui suit, on utilise toujours la norme du max.

\subsection{Estimations d'erreurs pour $f$ et ses dérivées partielles}

Si $f \in C^{3}(\Omega)$, on démontre les estimations d'erreurs suivantes, avec la notation $\left\|D^{3} f\right\|=\max \left\{\left\|D^{\gamma} f\right\|,|\gamma|=\right.$ $3\}$. Pour la fonction, on obtient :

$$
\left\|f-Q^{*} f\right\| \leq \frac{19}{48} h^{3}\left\|D^{3} f\right\| .
$$

Pour les dérivées partielles premières, avec les notations $D^{\varepsilon_{1}}=\frac{\partial}{\partial x}, D^{\varepsilon_{2}}=\frac{\partial}{\partial y}$, on a pour $k=1,2$. :

$$
\left\|D^{\varepsilon_{k}}\left(f-Q^{*} f\right)\right\|_{\Omega} \leq \frac{17}{4} h^{2}\left\|D^{3} f\right\| .
$$

Enfin, pour les dérivées secondes, avec les notations

$$
D^{2 \varepsilon_{1}}=\frac{\partial^{2}}{\partial x^{2}}, \quad D^{2 \varepsilon_{2}}=\frac{\partial^{2}}{\partial y^{2}}, \quad D^{\varepsilon_{1}+\varepsilon_{2}}=\frac{\partial^{2}}{\partial x \partial y},
$$

on obtient :

$$
\left\|D^{2 \varepsilon_{k}}\left(f-Q^{*} f\right)\right\|(k=1,2), \quad \text { et } \quad\left\|D^{\varepsilon_{1}+\varepsilon_{2}}\left(f-Q^{*} f\right)\right\| \leq \frac{41}{4} h\left\|D^{3} f\right\| .
$$


Les constantes indiquées ne sont pas optimales, mais elles en donnent un ordre de grandeur.

\subsection{Majorations des dérivées partielles d'ordres 1 et $\mathbf{2}$ de $Q^{*} f$}

On sait déjà que $\left\|Q^{*} f\right\| \leq \frac{3}{2}\|f\|$. On utilise les notations suivantes pour les dérivées partielles de $f$ et de son approximant $g=Q^{*} f$ :

$$
\begin{aligned}
& p=\frac{\partial f}{\partial x}, \quad q=\frac{\partial f}{\partial y}, \quad r=\frac{\partial^{2} f}{\partial x^{2}}, \quad s=\frac{\partial^{2} f}{\partial x \partial y}, \quad t=\frac{\partial^{2} f}{\partial y^{2}}, \\
& \pi=\frac{\partial g}{\partial x}, \quad \chi=\frac{\partial g}{\partial y}, \quad \rho=\frac{\partial^{2} g}{\partial x^{2}}, \quad \sigma=\frac{\partial^{2} g}{\partial x \partial y}, \quad \tau=\frac{\partial^{2} g}{\partial y^{2}}
\end{aligned}
$$

Comme $\pi$ et $\chi$ sont affines par triangle, il suffit de les majorer aux sommets de ces triangles, c'est à dire aux sommets et aux centres des carrés du réseau. On obtient les majorations suivantes :

$$
\|\pi\| \text { et }\|\chi\| \leq 2 \max \{\|p\|,\|q\|\} .
$$

De même, en calculant les dérivées partielles d'ordre 2 de $Q^{*} f$, constantes dans chaque triangle de la triangulation, on obtient les majorations suivantes :

$$
\|\rho\|,\|\sigma\|,\|\tau\| \leq \frac{25}{8} \max \{\|r\|,\|s\|,\|t\|\}
$$

On voit donc que l'approximation de $f$ par $Q^{*} f$ ne dilate pas trop la fonction et ses premières dérivées partielles. Pour les calculs, on utilise les résultats de la prépublication [47].

\subsection{Superconvergence en certains points}

Supposons que la fonction $f$ ait des d.p. d'ordre 4 bornées. Alors on observe [31] que l'on a, au centre, aux sommets et aux milieux des côtés d'un carré :

$$
f-Q^{*} f=O\left(h^{4}\right)
$$

Comme l'erreur est en général en $O\left(h^{3}\right)$, on a donc un phénomène de superconvergence en ces points. En revanche, pour les d.p. premières, on n'a pas ce phénomène aux mêmes points, mais il est probable qu'il se produise en certains points intérieurs du carré.

Pour les d.p. secondes, on a des résultats qui dépendent de l'orientation des triangles. Par exemple, pour les d.p. $\frac{\partial^{2}}{\partial x^{2}}$ sur les triangles Nord $(\mathrm{N})$ et Sud $(\mathrm{S})$, on a un phénomène de superconvergence :

$$
\left.(r-\rho)\right|_{N}=\left.(r-\rho)\right|_{S}=\frac{h^{2}}{24} D^{40} f(0)+O\left(h^{4}\right) .
$$

Sur les triangles Est(E) et Ouest (W), en posant $\left.e_{20}\right|_{E}=\left.(r-\rho)\right|_{E}$ et $\left.e_{20}\right|_{W}=\left.(r-\rho)\right|_{W}$, on obtient

$$
\left.e_{20}\right|_{E}=\frac{h}{2}\left[D^{30} f(0)-D^{12} f(0)\right]+O\left(h^{2}\right),\left.\quad e_{20}\right|_{W}=\frac{h}{2}\left[-D^{30} f(0)+D^{12} f(0)\right]+O\left(h^{2}\right),
$$

d'où une superconvergence pour la moyenne

$$
\frac{1}{2}\left(\left.e_{20}\right|_{E}+\left.e_{20}\right|_{W}\right)=O\left(h^{2}\right) .
$$

On a des résultats analogues pour les autres dérivées partielles secondes $\frac{\partial^{2}}{\partial y^{2}}$ et $\frac{\partial^{2}}{\partial x \partial y}$. 
Exemple 4.1. erreur sur le gradient de $f(x, y)=\exp \left(x^{2}+y^{2}\right)$.

On donne des résultats sur l'erreur maximale $|\operatorname{grad}(f-Q f)|$ respectivement au centre et aux sommets du carré $[-0.5,0.5]^{2}$ (on a ici $h=\frac{1}{N}$ ):

\begin{tabular}{|c|c|c|}
\hline$N$ & erreur centre & erreur sommets \\
\hline 8 & $2.61-04$ & $4.84-03$ \\
\hline 16 & $1.62-04$ & $2.15-03$ \\
\hline 32 & $7.24-05$ & $7.09-04$ \\
\hline 64 & $2.45-05$ & $2.04-04$ \\
\hline
\end{tabular}

\subsection{Un algorithme pour la détermination des points stationnaires de $g=Q^{*} f$}

Compte tenu des résultats ci-dessus sur l'approximation des dérivées de $f$ par celles de $Q^{*} f$, on propose l'algorithme suivant pour la recherche des points stationnaires de $Q^{*} f$ qui sont à priori voisins des points stationnaires de $f$.

(1) Calculer $\pi=\frac{\partial g}{\partial x}$ et $\chi=\frac{\partial g}{\partial y}$ aux sommets de la triangulation $\mathcal{T}$. Rappelons que ces deux fonctions sont continues et affines par morceaux, par conséquent elles sont entièrement déterminées par ces valeurs.

(2) Sélectionner le sous-ensemble $\mathcal{T}^{\prime} \subset \mathcal{T}$ des triangles dans lesquels la somme des signes de $\pi$ est \pm 1 .

(3) Sélectionner le sous-ensemble $\mathcal{T}^{\prime \prime} \subset \mathcal{T}^{\prime}$ des triangles dans lesquels la somme des signes de $\chi$ est \pm 1 .

(4) Dans chaque triangle de $\mathcal{T}^{\prime \prime}$, résoudre le système d'équations linéaires $\pi=\chi=0$.

On obtient ainsi les points stationnaires du quasi-interpolant $g=Q f$.

On peut alors faire une étude locale plus précise dans chaque triangle de la triangulation $\mathcal{T}^{\prime \prime}$ où se trouve un point stationnaire. Rappelons que les d.p. secondes $\rho, \sigma$ et $\tau$ de $g$ sont constantes dans chaque triangle, ce qui facilite cette étude.

Remarque 4.2. On peut évidemment permuter les rôles de $\pi$ et $\chi$ dans l'algorithme. Voici quelques exemples détaillés dans [30].

Exemple 4.3. $f(x, y)=\exp \left(x^{2}+y^{2}\right)$. On détecte facilement l'unique minimum de $f$ en $M_{0}=(0,0)$.

Exemple 4.4. $f(x, y)=\frac{3}{4} \exp \left(-\frac{1}{4}\left((9 x-2)^{2}+(9 y-2)^{2}\right)\right)+\frac{3}{4} \exp \left(-\left(\frac{1}{49}(9 x+1)^{2}+\frac{1}{10}(9 y+1)^{2}\right)\right)+\frac{1}{2} \exp \left(-\frac{1}{4}((9 x-\right.$ $\left.\left.7)^{2}+(9 y-3)^{2}\right)\right)-\frac{1}{5} \exp \left(-\left((9 x-4)^{2}+(9 y-7)^{2}\right)\right)$ (fonction de Franke [32]).

On détecte bien les 5 points stationnaires de $f$ dans le carré $[0,1]^{2}$ :

(1) le minimum local : $M_{0}=(0.461,0.783)$,

(2) les maxima locaux: $M_{1}=(0.207,0.209)$ et $M_{2}=(0.752,0.327)$,

(3) les points-selle ou dégénérés: $M_{3}=(0.561,0.256)$ et $\left.M_{4}=0.621,0.871\right)$.

Exemple 4.5. $f(x, y)=100\left((x+1)^{2}-(y+1)\right)^{2}+x^{2}$ (fonction de Rosenbrock [8]).

On détecte assez difficilement (à cause de la forme de la vallée) l'unique minimum de $g$ en $M_{0}=(0,0)$.

\section{Application : Formule de QUAdrature en Dimension 1 Associée À un QI QUADRATIQUE DISCRET}

Supposons que $I=[a, b]=[0, n h]$ et posons $t_{-2}=t_{-1}=a, t_{i}=i h$ pour $0 \leq i \leq n, t_{n+1}=t_{n+2}=b$, puis $\theta_{0}=a, \theta_{n+1}=b$, et $\theta_{i}=\frac{1}{2}\left(t_{i-1}+t_{i}\right)$, pour $1 \leq i \leq n$. Dans ce cas les $n+2$ B-splines $\left\{B_{k}, 0 \leq k \leq n+1\right\}$ telles que $\operatorname{supp}\left(B_{k}\right)=\left[t_{k-2}, t_{k+1}\right]$ forment une base de l'espace des splines quadratiques sur la partition uniforme de $I[1,5,12]$. Les données sont ici les valeurs $f_{i}=f\left(\theta_{i}\right), 0 \leq i \leq n+1$.

Le QI discret $Q^{*}$ s'écrit alors sous la forme :

$$
Q^{*} f=f_{0} B_{0}+\sum_{k=1}^{n} \mu_{k}^{*}(f) B_{k}+f_{n+1} B_{n+1}
$$


où les deux premières et les deux dernières formes coefficients sont :

$$
\mu_{0}^{*}(f)=f_{0}, \quad \mu_{1}^{*}(f)=\frac{h}{6}\left(-2 f_{0}+9 f_{1}-f_{2}\right), \quad \mu_{n}^{*}(f)=\frac{h}{6}\left(-f_{n-1}+9 f_{n}-2 f_{n+1}\right), \quad \mu_{n+1}^{*}(f)=f_{n+1},
$$

et, pour $2 \leq k \leq n-1$,

$$
\mu_{k}^{*}(f)=\frac{h}{8}\left(-f_{k-1}+10 f_{k}-f_{k+1}\right)
$$

Comme $Q^{*} f$ est une excellente approximation de $f$ sur $I$, on peut espérer une bonne approximation de l'intégrale $\mathcal{I}(f)=\int_{a}^{b} f$ par $\mathcal{I}_{2}(f)=\int_{a}^{b} Q^{*} f$. En intégrant $Q^{*} f$, on obtient la formule de quadrature suivante, pour $n \geq 5$ :

$$
\mathcal{I}_{2}(f)=\int_{a}^{b} Q^{*} f=\frac{h}{9} f_{0}+\frac{7 h}{8} f_{1}+\frac{73 h}{72} f_{2}+h \sum_{i=3}^{n-2} f_{i}+\frac{73 h}{72} f_{n-1}+\frac{7 h}{8} f_{n}+\frac{h}{9} f_{n+1} .
$$

On démontre le résultat d'erreur suivant [50] : il existe $C_{1}>0$ tel que, pour toute fonction $f$ assez régulière

$$
\mathcal{I}(f)-\mathcal{I}_{2}(f)=C_{1} h^{4} f^{(4)}\left(\gamma_{1}\right)+O\left(h^{5}\right), \quad \gamma_{1} \in I
$$

Cette formule est "complémentaire" de la formule de Simpson. Pour celle-ci, il existe $C_{2}>0$ tel que

$$
\mathcal{I}(f)-\mathcal{I}_{2}^{*}(f)=-C_{2} h^{4} f^{(4)}\left(\gamma_{2}\right), \quad \gamma_{2} \in I
$$

Bien que $\gamma_{1} \neq \gamma_{2}$ en général, en pratique on observe que les erreurs sont presque toujours de signes opposés. De plus, les constantes vérifient $0<C_{1}<C_{2}=\frac{1}{180}$.

Exemple 5.1. [52,53] : Comparons les résultats numériques obtenus en approchant les deux intégrales suivantes par les deux formules de quadrature $\mathcal{I}_{2}$ et $\mathcal{I}_{2}^{*}$ :

$$
\mathcal{I}\left(f_{1}\right)=\int_{-1}^{1} \frac{1}{1+16 x^{2}} d x \text { et } \mathcal{I}\left(f_{2}\right)=\int_{-1}^{1} e^{-x} \sin (5 \pi x) d x
$$

En posant respectivement $E_{2}(f)=\mathcal{I}(f)-\mathcal{I}_{2}(f), E_{2}^{*}(f)=\mathcal{I}(f)-\mathcal{I}_{2}^{*}(f)$, on obtient les résultats suivants (la notation $0.73(-9)$ signifie $\left.0.73 \times 10^{-9}\right)$ :

\begin{tabular}{|c|c|c|c|c|}
\hline$n$ & $E_{2}^{*}\left(f_{1}\right)$ & $E_{2}\left(f_{1}\right)$ & $E_{2}^{*}\left(f_{2}\right)$ & $E_{2}\left(f_{2}\right)$ \\
\hline 128 & $0.73(-9)$ & $-0.55(-9)$ & $0.14(-6)$ & $-0.11(-6)$ \\
\hline 256 & $0.45(-10)$ & $-0.33(-10)$ & $0.90(-8)$ & $-0.67(-8)$ \\
\hline 512 & $0.28(-11)$ & $-0.21(-11)$ & $0.56(-9)$ & $-0.41(-9)$ \\
\hline 1024 & $0.18(-12)$ & $-0.13(-12)$ & $0.73(-9)$ & $-0.52(-9)$ \\
\hline
\end{tabular}

On voit que les erreurs sont de signes opposés et ont des valeurs absolues du même ordre de grandeur.

\section{Calculs de déRivées et méthodes pseudo-SPeCtrales}

\subsection{Matrice de dérivation}

En dérivant $g=Q^{*} f$, et en posant $g_{i}^{\prime}=g\left(\theta_{i}\right), 0 \leq i \leq n+1$, on obtient les formules de dérivation suivantes. $h g_{0}^{\prime}=\frac{1}{3}\left(-8 f_{0}+9 f_{1}-f_{2}\right), \quad h g_{1}^{\prime}=\frac{1}{48}\left(-56 f_{0}+33 f_{1}+26 f_{2}-3 f_{3}\right), \quad h g_{2}^{\prime}=\frac{1}{48}\left(8 f_{0}-36 f_{1}+f_{2}+30 f_{3}-3 f_{4}\right)$, 
et des formules antisymétriques pour $h g_{n-1}^{\prime}, h g_{n}^{\prime}$ et $h g_{n+1}^{\prime}$. Pour $3 \leq i \leq n-2$, i.e. pour les points intérieurs, on a :

$$
h g_{i}^{\prime}=\frac{1}{16}\left(f_{i-2}-10 f_{i-1}+10 f_{i+1}-f_{i+2}\right) .
$$

Soit $\mathbf{f} \in \mathbb{R}^{n+2}$ et $\mathbf{g}^{\prime} \in \mathbb{R}^{n+2}$ les vecteurs de composantes respectives $f_{i}$ et $g_{i}^{\prime}$, pour $0 \leq i \leq n+1$. On peut écrire l'égalité matricielle :

$$
\mathbf{g}^{\prime}=\mathbf{D f}
$$

où $\mathbf{D}$ est la matrice de dérivation :

$$
\mathbf{D}=\frac{1}{h}\left[\begin{array}{ccccccccc}
-8 / 3 & 3 & -1 / 3 & 0 & 0 & 0 & \ldots & 0 & 0 \\
-7 / 6 & 11 / 16 & 13 / 24 & -1 / 16 & 0 & 0 & \ldots & 0 & 0 \\
1 / 6 & -3 / 4 & 1 / 48 & 5 / 8 & -1 / 16 & 0 & \ldots & 0 & 0 \\
0 & 1 / 16 & -5 / 8 & 0 & 5 / 8 & -1 / 16 & \ldots & 0 & 0 \\
\ldots & \ldots & \ldots & \ldots & \ldots & \ldots & \ldots & \ldots & \\
0 & 0 & \ldots & 1 / 16 & -5 / 8 & 0 & 5 / 8 & -1 / 16 & 0 \\
0 & 0 & \ldots & 0 & 1 / 16 & -5 / 8 & -1 / 48 & 3 / 4 & -1 / 6 \\
0 & 0 & \cdots & 0 & 0 & 1 / 16 & -13 / 24 & -11 / 16 & 7 / 6 \\
0 & 0 & \cdots & 0 & 0 & 0 & 1 / 3 & -3 & 8 / 3
\end{array}\right]
$$

\subsection{Erreur sur la dérivée}

Si l'on suppose $f$ suffisamment dérivable, on a les estimations d'erreur suivantes.

Pour $3 \leq i \leq n-2$, on obtient

$$
g_{i}^{\prime}-f_{i}^{\prime}=\frac{h^{2}}{24} f_{i}^{(3)}+O\left(h^{4}\right) .
$$

A l'origine et au premier point intérieur, on a respectivement :

$$
g_{0}^{\prime}-f_{0}^{\prime}=-\frac{h^{2}}{8} f_{0}^{(3)}+O\left(h^{3}\right), \quad \text { et } \quad g_{1}^{\prime}-f_{1}^{\prime}=\frac{h^{2}}{32} f_{1}^{(3)}+O\left(h^{3}\right) .
$$

(et des résultats analogues à la borne supérieure de l'intervalle).

Exemple 6.1. [52]: Considérons les deux fonctions $f_{1}(x)=\left(1+16 x^{2}\right)^{-1}$ et $f_{2}(x)=e^{-x} \sin (5 \pi x)$ sur l'intervalle $I=[-1,1]$. Pour $p=1,2$, on pose $\varepsilon_{p}=\max \left|f_{p}^{\prime}\left(\theta_{i}\right)-g_{p}^{\prime}\left(\theta_{i}\right)\right|$ et $\varepsilon_{p}^{*}=\max \left|f_{p}^{\prime}\left(\theta_{i}\right)-\delta f_{p}\left(\theta_{i}\right)\right|$, où $\delta f_{p}\left(\theta_{i}\right)$ est la différence centrée de $f_{p}$ au point $\theta_{i}$ (avec les modifications usuelles aux extrémités de I). On obtient les résultats suivants :

\begin{tabular}{|c|c|c|c|c|}
\hline$n$ & $\varepsilon_{1}$ & $\varepsilon_{1}^{*}$ & $\varepsilon_{2}$ & $\varepsilon_{2}^{*}$ \\
\hline 64 & $1.4(-2)$ & $4.8(-2)$ & $1.6(-2)$ & $4.6(-2)$ \\
\hline 128 & $3.1(-3)$ & $1.2(-2)$ & $3.7(-3)$ & $1.2(-2)$ \\
\hline 256 & $7.7(-4)$ & $3.0(-3)$ & $8.7(-4)$ & $2.9(-3)$ \\
\hline 512 & $1.9(-4)$ & $7.6(-4)$ & $2.1(-4)$ & $7.2(-3)$ \\
\hline 1024 & $4.7(-5)$ & $1.9(-4)$ & $5.2(-5)$ & $1.8(-4)$ \\
\hline
\end{tabular}

On voit que les erreurs sont en $O\left(h^{2}\right)$, mais celles obtenues par le QI sont 3 à 4 fois plus faibles que celles fournies par la différence centrée. 


\subsection{Méthode pseudo-spectrale}

Supposons que l'on veuille résoudre le problème de Dirichlet homogène suivant :

$$
\left\{\begin{array}{l}
u^{\prime \prime}(x)=f(x), \\
u(-1)=u(1)=0 .
\end{array} \quad \text { pour } x \in I=(-1,1)\right.
$$

où l'on suppose par exemple $f$ continue. Il est facile de montrer (Cf $[6,13])$ que le vecteur $\mathbf{u} \in \mathbb{R}^{n}$ des valeurs approchées de $u\left(\theta_{i}\right)$, pour $1 \leq i \leq n$, est solution du système linéaire

$$
\tilde{\mathbf{D}} \mathbf{u}=\mathbf{f}
$$

où $\mathbf{f}$ est le vecteur de composantes $f\left(\theta_{i}\right)$ et $\tilde{\mathbf{D}} \in \mathbb{R}^{n \times n}$ la sous-matrice formée des lignes et colonnes numérotées de 1 à $n$ de la matrice $\mathbf{D}^{2}$.

Exemple 6.2. Choisissons $f_{1}(x)=12 x^{2}-4$ qui correspond à la solution $u_{1}(x)=\left(1-x^{2}\right)^{2}$, et $f_{2}(x)=e^{4 x}$ qui correspond à la solution $u_{2}(x)=\left(e^{4 x}-\sinh (4) x-\cosh (4)\right) / 16$. Pour $p=1,2$, on pose respectivement $\varepsilon_{p}=\max \left\{\left|\mathbf{u}_{p}(i)-u_{p}\left(\theta_{i}\right)\right|, 1 \leq i \leq n\right\}$, où $\tilde{\mathbf{D}} \mathbf{u}_{p}=\mathbf{f}_{p}$. On obtient les résultats suivants :

\begin{tabular}{|c|c|c|c|c|c|}
\hline$n$ & 20 & 40 & 60 & 80 & 100 \\
\hline$\varepsilon_{1}$ & $1.0(-2)$ & $2.5(-3)$ & $1.1(-3)$ & $6.0(-4)$ & $4.0(-4)$ \\
\hline$\varepsilon_{2}$ & $3.4(-2)$ & $7.8(-3)$ & $3.4(-3)$ & $1.9(-3)$ & $1.2(-3)$ \\
\hline
\end{tabular}

Les deux lignes font apparaître clairement une erreur en $O\left(h^{2}\right)$. On peut améliorer ces résultats de façon significative en choisissant des subdivisions adaptées.

\section{Applichtion : ReCherche de zÉros}

On peut approcher les zéros d'une fonction $f$ par ceux du QI spline $g=Q^{*} f$ qui sont calculables exactement car $g$ est quadratique par morceaux.

Exemple 7.1. : Approximation des zéros du polynôme de Legendre $f=P_{8}$ sur $I=[-1,1]$ par ceux du $Q I g$. Les zéros de $P_{8}$ sont $\left\{ \pm x_{1}, \pm x_{2}, \pm x_{3}, \pm x_{4}\right\}$, avec $x_{1} \approx 0.1834, x_{2} \approx 0.5255, x_{3} \approx 0.7967, x_{4} \approx 0.9603$.

Voici les erreurs sur les zéros (en valeur absolue) pour différentes valeurs de $n$.

\begin{tabular}{|c|c||c|c|c|c|c|}
\hline$n$ & 8 & 16 & 32 & 64 & 128 & 256 \\
\hline$e_{1}$ & $1.5-2$ & $5.4-4$ & $4.3-5$ & $1.3-5$ & $2.0-6$ & $3.6-8$ \\
\hline$e_{2}$ & $2.7-2$ & $3.8-3$ & $2.1-4$ & $1.2-5$ & $1.3-6$ & $3.6-7$ \\
\hline$e_{3}$ & $1.1-1$ & $1.2-2$ & $5.6-4$ & $4.3-5$ & $3.3-6$ & $9.2-8$ \\
\hline$e_{4}$ & $4.4-2$ & $6.6-3$ & $3.1-4$ & $9.3-5$ & $4.5-6$ & $5.4-7$ \\
\hline
\end{tabular}

\section{GÉnÉRAlisations et DÉveloppements}

Le présent survol n'est qu'une introduction aux QI quadratiques en dimensions 1 et 2, sur des partitions uniformes, et à quelques applications. Pour les extensions à d'autres degrés et à des partitions non uniformes, on peut consulter par exemple les livres généraux [1-5,12], les survols [16,17,48,52] et les articles [19,25,27,28, 30,34-36,38,56]. Plus récemment, nous avons défini des QI à deux variables sur des triangulations quelconques de type Powell-Sabin [31,39,40,44] en utilisant les B-splines introduites par Dierckx [29]. 


\section{BibliographiE}

\subsection{Abréviations : journaux et éditeurs}

ACHA=Appl. \& Comput. Harmonic Anal. (journal).

$\mathrm{AiCM}=$ Advances in Comput. Math. (journal). AKP=A.K. Peters, Wellesley.

$\mathrm{AMS}=$ American Mathematical Society. AP=Academic Press, London, New-York.

ATA =Approximation Theory \& Applications, ou Analysis in Theory \& Applications (journal).

$\mathrm{BC}=$ Brooks $/$ Cole Publ. BV=Birkhäuser Verlag, Basel.

$\mathrm{CA}=$ Constr. Approx. (journal). CAGD=Comput. Aided Geom. Design. (journal).

$\mathrm{CUP}=$ Cambridge University Press. IMAJNA=IMA J. Numer. Anal. (journal).

IRMAR $=$ Institut de Recherche Mathématique de Rennes.

ISNM=International Series on Numerical Mathematics (BV).

JAT=Journal of Approx. Theory. (journal). JCAM=J. Comput. Appl. Math. (journal).

JWS=John Wiley \& Sons, New-York. $\mathrm{K}=$ Kluwer, Dordrecht.

$M^{2} A N=$ Math. Modelling and Numer. Anal. (journal).

$\mathrm{MC}=$ Math. of Comput. (journal). OUP=Oxford University Press.

SIAM=Society for Industrial and Applied Mathematics, Philadelphia.

SIAM JNA=SIAM J. Numer. Anal. (journal). SV=Springer-Verlag, Berlin.

Livres de [1] à [13] et les articles de [14] à [58].

\section{References}

[1] B.D. Bojanov, H.A. Hakopian, A.A. Sahakian, Spline functions and multivariate interpolation, K 1993.

[2] C. de Boor, K. Höllig, S. Riemenschneider : Box-splines. SV 1993.

[3] C.K. Chui : Multivariate splines, CBMS-NSF Regional Conference Series in Applied Mathematics, vol. 54, SIAM 1988.

[4] W. Cheney, W. Light : A course in approximation theory. BC 2000.

[5] R. DeVore, G.G. Lorentz : Constructive Approximation. SV 1993.

[6] B. Fornberg : A practical guide to pseudospectral methods. CUP 1998.

[7] W. Gautschi : Orthogonal polynomials. OUP 2004.

[8] Ph.E. Gill, W. Murray, M.H. Wright : Practical Optimization. AP 1997.

[9] M.J.D. Powell : Approximation theory and methods, CUP 1980.

[10] P. Sablonnière : Bases de Bernstein et approximants splines. Thèse d'état, Lille, 1982.

[11] I.J. Schoenberg : Cardinal spline interpolation. Regional Conference Series in Applied Mathematics, vol. 12 , SIAM 1973.

[12] L.L. Schumaker : Spline functions : basic theory. JWS 1981.

[13] L. Trefethen : Spectral methods in Matlab, SV 2000.

[14] H. Akima : On estimating partial derivatives for bivariate interpolation of scattered data. Rocky Mountains J. Math. 14, No 1 (1984) 41-75.

[15] E. Arge, M. Daehlen : Grid point interpolation on finite regions using $C^{1}$ box splines. SIAMJNA 29, No 4 (1992) $1136-1153$.

[16] C. de Boor : Splines as linear combinations of B-splines. In Approximation Theory II, G.G. Lorentz et al. (eds), AP (1976) $1-47$.

[17] C. de Boor : Quasi-interpolants and approximation power of multivariate splines. In Computation of curves and surfaces, W. Dahmen et al. (eds), K (1990) 313-345.

[18] C. de Boor, T. Lyche, L.L. Schumaker : On calculating with B-splines II, Integration. in Numerische Methoden der Approximationstheorie, (Eds. L. Collatz \& al. ), ISNM Vol. 30, BV (1976) 123-146.

[19] C.K. Chui, M.J. Lai : A multivariate analog of Marsden's identity and a quasi-interpolation scheme. CA 3 (1987) 111-122.

[20] C.K. Chui, M.J. Lai : Computation of box splines and B-splines on triangulations of nonuniform rectangular partitions. ATA 3 (1987) 37-62.

[21] C.K. Chui, L.L. Schumaker, R.H. Wang, On spaces of piecewise polynomials with boundary conditions III. Type II triangulations. In: Canadian Mathematical Society Conference Proceedings, Vol. 3, AMS (1983), 67-80.

[22] C.K. Chui, R.H. Wang, On a bivariate B-spline basis, Scientia Sinica XXVII, No 11 (1984), $1129-1142$.

[23] C.K. Chui, R.H. Wang, Concerning $C^{1}$ B-splines on triangulations of nonuniform rectangular partitions, ATA 1 (1984), 11-18.

[24] M. Daehlen, T. Lyche : Bivariate interpolation with quadratic box-splines. MC 51, No 183 (1988) $219-230$.

[25] C. Dagnino, P. Lamberti : Some performances of local bivariate quadratic $C^{1}$ quasi-interpolating splines on non-uniform type-2 triangulations. JCAM 173 (2005) 21-37. 
[26] C. Dagnino, P. Lamberti : Spline quasi-interpolants with boundary conditions on criss-cross triangulations. To appear in Curves and Surfaces, Avignon 2006, T. Lyche et al. (eds), Nashboro Press, 2007.

[27] C. Dagnino, P. Sablonnière : Error analysis for quadratic spline quasi-interpolants on non-uniform criss-cross triangulations of bounded rectangular domains. Article soumis.

[28] V. Demichelis, P. Sablonnière : Numerical integration by spline quasi-interpolants in three variables. To appear in Curves and Surfaces, Avignon 2006, T. Lyche et al. (eds), Nashboro Press, 2007.

[29] P. Dierckx : On calculating normalized Powell-Sabin B-splines. CAGD 15 (1997) 61-78.

[30] F. Foucher, P. Sablonnière : Approximating partial derivatives of first and second order by quadratic spline quasi-interpolants. Congrès MAMERN, Oujda, Maroc, 9-11 mai 2005. Prépublication IRMAR 06-25 (2006).A paraître.

[31] F. Foucher, P. Sablonnière : Superconvergence properties of some bivariate $C^{1}$ quadratic spline quasi-interpolants. To appear in Curves and Surfaces, Avignon 2006, T. Lyche et al. (eds), Nashboro Press, 2007.

[32] R. Franke : Scattered data interpolation : tests of some methods. MC 38 (1982) 181-200.

[33] W. Gautschi, L. Gori, F. Pitolli : Gauss quadrature for refinable weight functions. ACHA 8 (2000) 249-257.

[34] T.N.T. Goodman: Total positivity and the shape of curves. In Total positivity and its applications. (Eds. M. Gasca \& C.A. Micchelli), K (1996) 157-186.

[35] T.N.T. Goodman, S.L. Lee : Spline approximation operators of Bernstein-Schoenberg type in one and two variables. JAT 33 (1981) 248-263.

[36] T.N.T. Goodman, A. Sharma : A modified Bernstein-Schoenberg operator. In Constructive theory of functions '87, (Ed. Bl. Sendov), Bulgarian Academy of Science, Sofia (1988) 166-173.

[37] F. Jeeawock-Zedek : Interpolation de Lagrange par des splines quadratiques sur un quadrilatère de $\mathbb{R}^{2} . M^{2} A N$ 26, No 5 (1992) 575-594.

[38] T. Lyche, L.L. Schumaker : Local spline approximation methods. JAT 15 (1975) 294-325.

[39] C. Manni, P. Sablonnière : Quadratic spline quasi-interpolants on Powell-Sabin partitions. AiCM 1-3 (2007), 283-304.

[40] C. Manni : Shape control in Powell-Sabin quasi-interpolation. In Algorithms for Approximation V, a. Iske and j. Levesley (eds), SV (2006), 219-240.

[41] E. Neumann : Moments and Fourier transforms of B-splines. JCAM 7 (1981), 51-62.

[42] J.L. Phillips, R.J. Hanson : Gauss quadrature rules with B-spline weight functions. MC 28 (1974) 18.

[43] M.J.D. Powell : Piecewise quadratic surface fitting for contour plotting. In Software for numerical mathematics, D.J. Evans (ed), AP (1974) 253-271.

[44] M.J.D. Powell, M.A. Sabin : Piecewise quadratic approximation on triangles. ACM Trans. Math. Software 3 (1977) 316-325.

[45] P. Sablonnière : Bernstein-Bézier methods for the construction of bivariate spline approximants, CAGD 2 (1985), 29-36.

[46] P. Sablonnière : Error bounds for Hermite interpolation by quadratic splines on an alpha-triangulation. IMAJNA 7 (1987) 495-508.

[47] P. Sablonnière : BB-coefficients of basic bivariate quadratic splines on rectangular domains with uniform criss-cross triangulations. Prépublication IRMAR 02-56, Décembre 2002.

[48] P. Sablonnière : On some multivariate quadratic spline quasi-interpolants on bounded domains, in: Modern developments in multivariate approximation (Eds. Hausmann W., Jetter K., Reiner M. and Stöcker J.), ISNM Vol. 145, BV ( 2003) $263-278$.

[49] P. Sablonnière : Quadratic spline quasi-interpolants on bounded domain of $R^{d}, d=1,2,3$, Rend. Sem. Mat. Univ. Pol. Torino 61 (2003), 229-246.

[50] P. Sablonnière : Quadratic B-splines on non uniform criss-cross triangulations of bounded rectangular domains of the plane. Prépublication IRMAR 03-14, Mars 2003.

[51] P. Sablonnière : Univariate spline quasi-interpolants and applications to numerical analysis. Rend. Sem. Mat. Univ. Pol. Torino 63 No 2 (2005) 107-118.

[52] P. Sablonnière : Recent progress on univariate and multivariate polynomial and spline quasi-interpolants. In Trends and applications in constructive approximation, M.G. de Bruijn, D.H. Mache and J. Szabados (eds), ISNM Vol. 151, BV (2005) 229-245.

[53] P. Sablonnière : Refinement equation and subdivision algorithm for quadratic B-splines on non-uniform criss-cross triangulations. In Proceedings of the International Conference Wavelets and splines, July 3-8, 2003. St Petersburg University Press (2005) 84-102.

[54] P. Sablonnière : A quadrature formula associated with a quadratic spline quasi-interpolant. Prépublication IRMAR 05-40, Décembre 2005. Article soumis.

[55] P. Sablonnière, F. Jeeawock-Zedek : Hermite and Lagrange interpolation by quadratic splines on nonuniform criss-cross triangulations. In Curves and surfaces, P.J. Laurent et al. (eds), AKP (1991) 445-452.

[56] P. Sablonnière, D. Sbibih : Spline integral operators exact on polynomials. ATA 10, No 3 (1994) 56-73.

[57] I.J. Schoenberg : On variation diminishing approximation methods. In On numerical approximation, (Ed. R.E. Langer), University of Wisconsin Press, Madison (1959) 249-274.

[58] P.B. Zwart : Multivariate splines with nondegenerate partitions. SIAM J. Numer. Anal. 10 (1973) 665-673. 